\title{
Energetic electron acceleration by unsteady magnetic reconnection
}

\author{
H. S. Fu ${ }^{1,2 \star}$, Yu. V. Khotyaintsev' ${ }^{1}$ A. Vaivads ${ }^{1}$, A. Retinò ${ }^{3}$ and M. André
}

\begin{abstract}
The mechanism that produces energetic electrons during magnetic reconnection is poorly understood. This is a fundamental process responsible for stellar flares ${ }^{1,2}$, substorms ${ }^{3,4}$, and disruptions in fusion experiments ${ }^{5,6}$. Observations in the solar chromosphere ${ }^{1}$ and the Earth's magnetosphere ${ }^{7-10}$ indicate significant electron acceleration during reconnection, whereas in the solar wind, energetic electrons are absent". Here we show that energetic electron acceleration is caused by unsteady reconnection. In the Earth's magnetosphere and the solar chromosphere, reconnection is unsteady, so energetic electrons are produced; in the solar wind, reconnection is steady $^{12}$, so energetic electrons are absent ${ }^{11}$. The acceleration mechanism is quasi-adiabatic: betatron and Fermi acceleration in outflow jets are two processes contributing to electron energization during unsteady reconnection. The localized betatron acceleration in the outflow is responsible for at least half of the energy gain for the peak observed fluxes.
\end{abstract}

Observations of the Earth's magnetotail ${ }^{7-9}$ and magnetopause ${ }^{10}$, the solar chromosphere ${ }^{1}$, and the Galactic centre ${ }^{13}$ reveal that electrons can be accelerated to several times their thermal energy during and following reconnection. Several mechanisms have been proposed in the frame of steady reconnection to explain the generation of such electrons at the reconnection $\mathrm{X}$-line-Joule heating ${ }^{14}$, reconnection electric fields ${ }^{15}$, and parallel electric fields ${ }^{16,17}$. However, observations in the solar wind ${ }^{11}$, where reconnection is typically steady ${ }^{12}$, show no substantial electron acceleration, suggesting that a model involving unsteady reconnection and/or multiple acceleration sites is needed. The latter scenario assumes several reconnection points and magnetic island ${ }^{18-20}$ near the small diffusion region, however the feasibility of this assumption is debatable ${ }^{8,18}$; also, the acceleration is limited by the back pressure of electrons ${ }^{18}$. So far, the connection between electron acceleration and unsteady reconnection has not been established.

Dipolarization fronts (also named jet fronts) in the Earth's magnetotail together with the time-varying speed of plasma jets have been identified as the signatures of unsteady reconnection ${ }^{21,22}$. The energetic electrons around jet fronts ${ }^{23,24}$ and magnetic pileup regions $s^{7,25}$, therefore, can be used to investigate acceleration mechanisms during unsteady reconnection. The ESA spacecraft mission Cluster, orbiting the Earth's magnetosphere for about one solar cycle (2000-now), provides a unique opportunity to address this issue, as it encounters the jet fronts frequently when moving through the magnetotail.

On 23 August 2006, Cluster 1 was in the Earth's plasma sheet, located at $[-17.2,-6.9,0.0]$ Earth radii $\left(R_{\mathrm{E}}\right)$ in geocentric solar magnetospheric (GSM) coordinates (Fig. 1). It observed a jet front at 07:00:22 Ut (Fig. 2b), when the magnetic field $B_{z}$ component jumped from 3 to $11 \mathrm{nT}$. Ahead of the jet front, $B_{z}$ was relatively stable, with a value of $4 \mathrm{nT}$; whereas behind the jet front, $B_{z}$ was strong, with a value of $10 \mathrm{nT}$ to about 07:01:06 UT, after which $B_{z}$ recovered to the same small value as in the plasma sheet. Two reconnection jets with maximum speeds of $750 \mathrm{~km} \mathrm{~s}^{-1}$ and $550 \mathrm{~km} \mathrm{~s}^{-1}$ were observed inside the flux pile-up region (FPR, Fig. 2c). They were separated by about $25 \mathrm{~s}$. Each jet included a rising speed portion (yellow) and a falling speed portion (green). Energetic electrons, up to 127 kilo-electronvolts $(\mathrm{keV})$, were observed inside each reconnection jet (Fig. 2e), with the highest fluxes corresponding to the rising speed portion of the jets whereas the lowest fluxes correspond to the falling speed portion. Electron phase space densities (PSD) of four regions, indicated by the horizontal black bars between Fig. 2e,f, are shown in Fig. 3a. These electrons almost follow a common power law distribution PSD $\propto \varepsilon^{-\gamma}$, where $\varepsilon$ is the energy of the electrons and $\gamma=4.2$ is a typical value in the Earth's magnetotail ${ }^{7,9}$, suggesting that the acceleration process is quasi-adiabatic.

Figure $2 \mathrm{f}$ shows the pitch angle distribution of the energetic electrons. During the rising speed portion of the plasma jets the electrons tend to be perpendicular to the magnetic field, whereas during the falling speed portion of the jets the electrons tend to be parallel to the field, as reported recently ${ }^{24,26}$. This characteristic is clearer in Fig. 2g, where the PSDs of electrons are shown.

The two plasma jets (Fig. 2c) inside the FPR are attributed to unsteady reconnection, which may be externally driven, for example by perturbations near the magnetopause $e^{22,27}$, or internally driven by turbulence and instabilities inside the ion diffusion region $^{28,29}$. A perturbation near the magnetopause can lead to a time-varying inflow speed near the X-line (Fig. 1) and then a significant change of the local Alfven speed ${ }^{22}$ that defines the outflow jet speed. Also intrinsic instabilities may cause transient changes in the reconnection rate ${ }^{29}$, leading to drastic changes in the jet speed ${ }^{28}$ and the formation of dipolarization fronts.

The highest electron fluxes are found in the direction perpendicular to the magnetic field in the rising speed portion of the jets. We interpret these fluxes as being due to localized betatron acceleration of electrons in the outflow region, where the frozen-in condition is satisfied. During the jet speed rising, the trailing part of the flux tube is moving towards the leading part of the flux tube, so that the volume of flux tubes contracts (see the opposite arrows in Fig. 1c). In this situation, the local magnetic field is enhanced and betatron acceleration occurs ${ }^{24}$. During the jet speed falling, the trailing part of the tube is moving away from the leading part of the tube; the volume of flux tubes expands, so that the localized betatron cooling occurs. This explains the low fluxes of

\footnotetext{
${ }^{1}$ Swedish Institute of Space Physics, Box 537, Uppsala 75121, Sweden, ${ }^{2}$ Space Science Institute, School of Astronautics, Beihang University, 37 Xueyuan Road, Haidian District, Beijing 100191, China, ${ }^{3}$ Laboratoire de Physique des Plasmas, CNRS-Ecole Polytechnique-Université Pierre et Marie Curie-Université Paris-Sud, Route de Saclay, F-91128 Palaiseau, France. *e-mail: huishan@irfu.se
} 

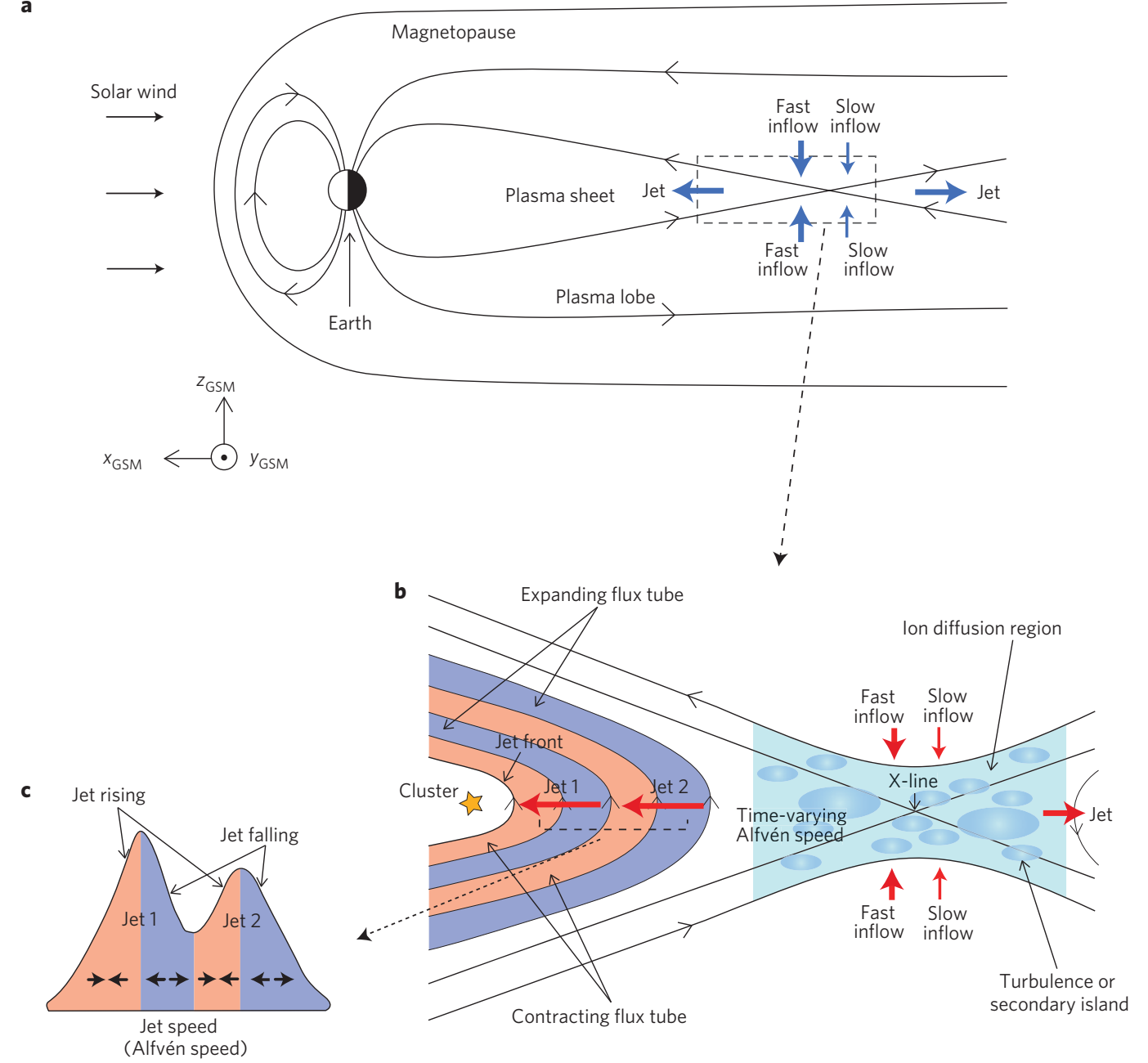

Figure 1 | Sketch of unsteady reconnection in the Earth's magnetotail. a, Earth's magnetosphere in the noon-midnight plane, showing a convergence of magnetic field lines in the nightside plasma sheet that is characterized by high plasma beta (ratio of thermal pressure to magnetic pressure). During the 'break' and 'reconnection' of magnetic field lines, magnetic energy is transformed to kinetic energy of the particles. Owing to the change of external conditions (for example, perturbations near the magnetopause ${ }^{22,27}$ ), the inflow can be fast or slow, and also the local Alfvén speed may vary, resulting in changes of the outflow jet speed ${ }^{22}$. b. Magnified view of the ion diffusion region and the reconnection jet. Secondary islands and turbulence around the $X$-line have been found in simulations ${ }^{18,20,28,29}$. Changes in the inflow speed may produce the two reconnection jets ${ }^{22}$ that have been observed by Cluster in the downstream region at $[-17.2,-6.9,0.0] R_{\mathrm{E}}$. Turbulence and secondary islands can also affect the reconnection process ${ }^{28,29}$, and then produce the two reconnection jets as well. c, Schematic showing the speeds of the two reconnection jets. Each jet consists of a rising speed portion (orange) and a falling speed portion (blue). In the rising speed portion of the jets, the flux tubes contract and localized betatron acceleration of electrons occurs ${ }^{24}$; in the falling speed portion, the flux tubes expand and localized betatron cooling of electrons is expected.

electrons in the perpendicular direction (Fig. 2f). The localized betatron mechanism we propose here is different from the global betatron mechanism, which accelerates electrons during the $\nabla B$ drift opposite to a finite electric field ${ }^{25}$.

If electrons bounce along the magnetic field line and simultaneously the length of the field line is shrinking (for example, the earthward-moving flux tubes in the Earth's magnetotail or the downward-moving flux tubes in the solar chromosphere), Fermi acceleration is expected. This mechanism is observed in the present event as the field-aligned fluxes of the energetic electrons in the FPR (07:00:26-07:01:03 UT) are all larger than those in the plasma sheet (07:00:18 UT). At 07:00:26 UT and 07:00:43-07:00:47 UT, the field-aligned fluxes increase by a small factor, meaning that Fermi acceleration due to magnetic field line shrinkage is not prominent in this event and that Cluster was located close to the X-line.

During the short time ( $2 \mathrm{~min}$ ) of this event, Fermi acceleration due to magnetic field line shrinkage should be same inside the whole FPR. However, we find that the field-aligned fluxes at 07:00:30-
07:00:39 UT are clearly larger than those at 07:00:26 UT (Fig. 2f). This implies other field-aligned acceleration processes near the reconnection X-line (for example, localized Fermi acceleration by contracting magnetic islands $\left.{ }^{18}\right)$. These processes occur primarily during the decline of the plasma jets. Although our analysis does not reveal the details of this acceleration mechanism, we can still conclude that it is an adiabatic process as the power law index inside the whole FPR is almost a constant $\gamma=4.2$ (Fig. 3a).

The quantitative estimation of particle acceleration (see Methods) is shown in Fig. 3b-g. One can see that the factor $f_{\perp}=1.3$ during the jet speed rising (Fig. $3 c, e$ ) and the factor $f_{\|}=1.4$ during the jet speed falling (Fig. 3d) are sufficient to produce the observed fluxes in Fig. 2f. During the jet speed falling (Fig. 3d,f), the factor $f_{\perp}$ is somewhat smaller than one, indicating that betatron cooling of electrons occurs. We compare the PSDs during the jet speed rising (Fig. 3c,e) to the average PSDs (Fig. 3g) inside the whole FPR (07:00:26-07:01:03 UT), to examine the contribution of localized betatron acceleration in this event. We find that the perpendicular 


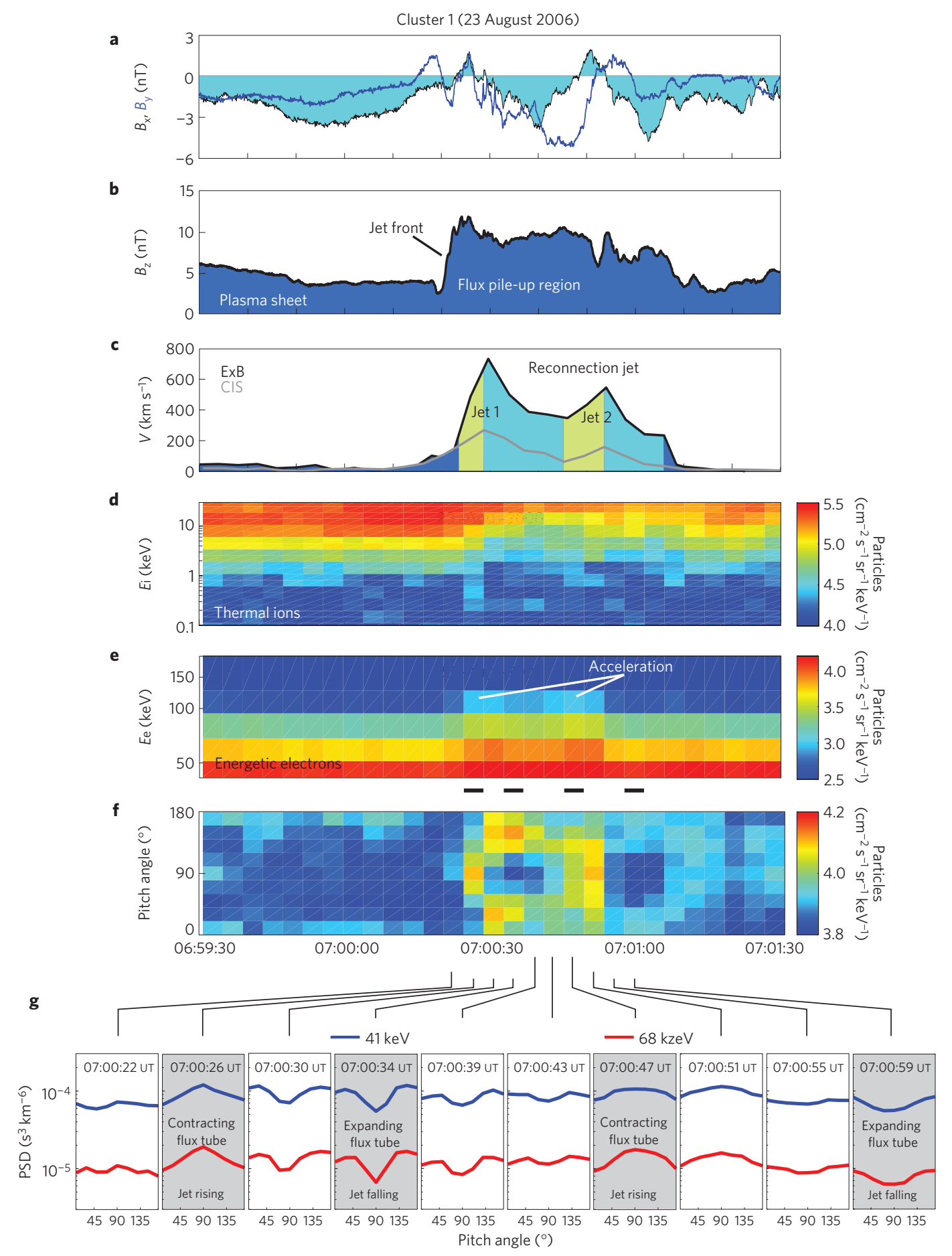

Figure 2 | Cluster 1 observations of electron acceleration by unsteady reconnection. a, The magnetic field $B_{x}$ (black) and $B_{y}$ (blue) components in GSM coordinates. $\mathbf{b}$, The magnetic field $B_{z}$ component in GSM coordinates. The jet front is located at the $B_{z}$ jump. In front of it is the plasma sheet, while behind it is the flux pile-up region (FPR; ref. 24). $\mathbf{c}$, The bulk speed of the plasma flow measured by CIS (grey) and derived from $\mathbf{E} \times \mathbf{B}$ (black). CIS is an ion detector on the Cluster spacecraft, covering the energy range from $5 \mathrm{eV}$ to $32 \mathrm{keV}$. $\mathbf{E}$ and $\mathbf{B}$ are measured by the EFW and FGM instruments. The speed from CIS is significantly smaller than that from $\mathbf{E} \times \mathbf{B}$, because some of the energetic ions are beyond the energy range of CIS. Apart from the magnitude, the speeds from CIS and $\mathbf{E} \times \mathbf{B}$ follow the same trend. Two reconnection jets are observed inside the FPR. $\mathbf{d}$, Differential particle flux (DPF) of the ions measured by CIS. Inside the FPR, part of the ion distribution is beyond the upper energy threshold. e, DPF of electrons from 40 to 200 keV, measured by the RAPID instrument. f, Pitch angle (an angle between the electron speed and the magnetic field line, with $90^{\circ}$ denoting the perpendicular motion of electrons and $0^{\circ} / 180^{\circ}$ denoting the parallel/anti-parallel motion of electrons) distribution of the 41 and $68 \mathrm{keV}$ electrons. For other energy channels, the pitch angle distribution is not available in this event owing to the instrument measurement mode. The enhancement of DPF around $90^{\circ}$ pitch angle indicates perpendicular acceleration of electrons, whereas the enhancement of DPF around $0^{\circ} / 80^{\circ}$ pitch angle indicates field-aligned acceleration. $\mathbf{g}$, Phase space densities (PSDs) of the 41 and $68 \mathrm{keV}$ electrons, measured from 07:00:22 to 07:00:59 UT. The grey shadow frames highlight the PSDs in the rising (07:00:26, 07:00:47 UT) and falling (07:00:34, 07:00:59 UT) speed parts of the reconnection jets. 

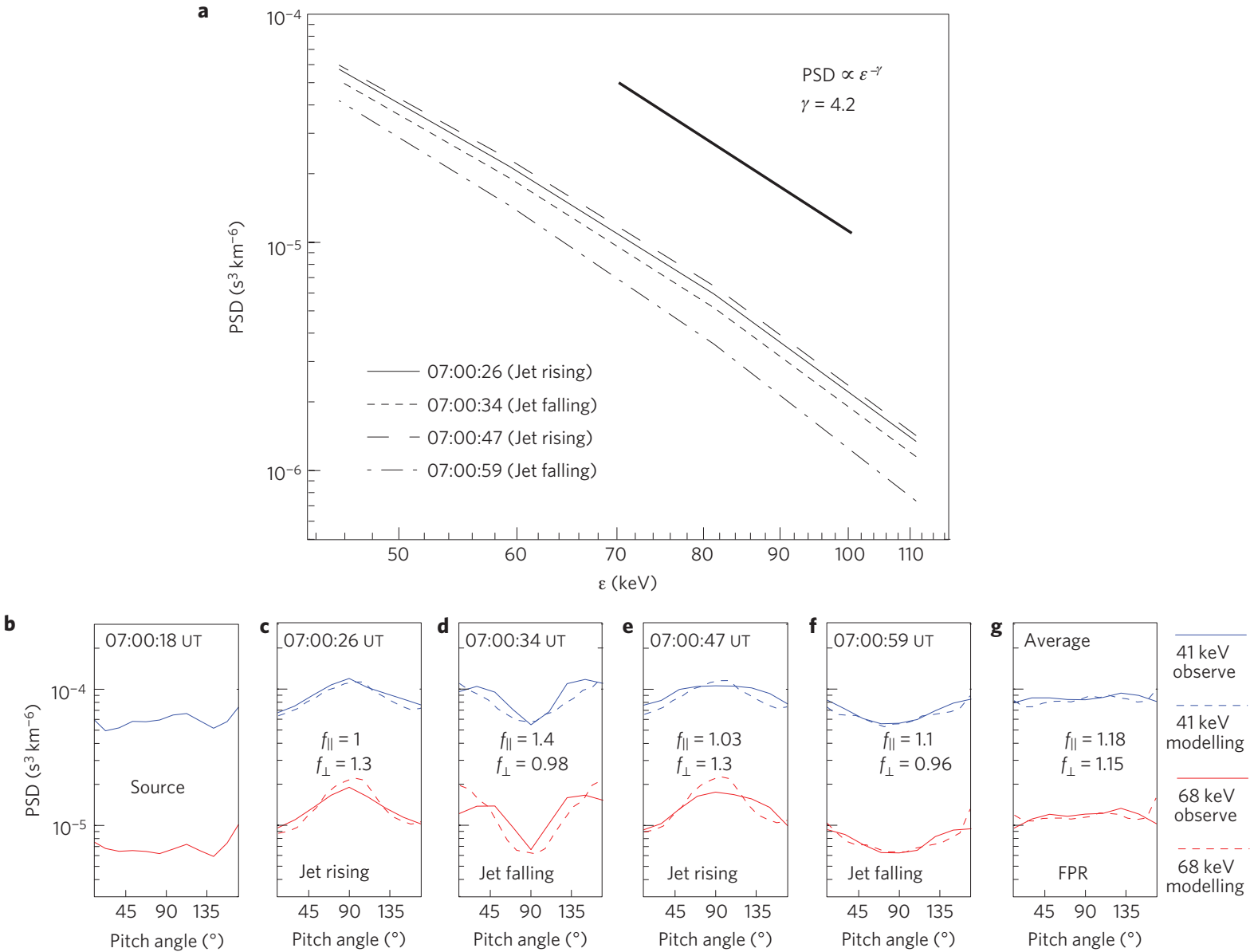

Figure 3 | Modelling of electron acceleration by unsteady reconnection. a, Phase space densities (PSDs) of the $45-111$ keV electrons averaged over all viewing directions. They are measured at 07:00:26, 07:00:34, 07:00:47, and 07:00:59 UT respectively, and correspond to the horizontal black bars between Fig. 2e,f. Measurements at these times follow a common power law distribution with the index $\gamma=4.2$ (thick solid line), indicating that the electron acceleration is quasi-adiabatic. b-g, PSDs of the 41 and $68 \mathrm{keV}$ electrons, shown as a function of pitch angle. PSDs in $\mathbf{b}$ were observed in the unperturbed plasma sheet; they are used as the source population when estimating electron acceleration. PSDs in $\mathbf{g}$ are obtained by averaging the PSDs inside the whole FPR (07:00:26-07:01:03 UT). In c-g, solid lines represent the Cluster observations, while dashed lines represent the modelling of electron acceleration. $f_{\|}$and $f_{\perp}$ are the factors of parallel $\left(f_{\|}\right)$and perpendicular $\left(f_{\perp}\right)$ acceleration. To estimate the value of $f_{\|}$and $f_{\perp}$, we fit a model to the observations.

acceleration in Fig. $3 c$,e $\left(f_{\perp}=1.3\right)$ is about twice as large as the perpendicular acceleration in Fig. $3 g\left(f_{\perp}=1.15\right)$, meaning that localized betatron acceleration contributes at least $50 \%$ to the peak particle energization. Therefore, the whole acceleration process can be decomposed into two steps. First, the electrons from the source (Fig. 3b) are accelerated at the X-line by less than the average factor $\left(f_{\perp}=1.15\right)$ in the outflow. Then, the electrons are further accelerated in the outflow region due to localized betatron and Fermi mechanisms.

Our findings can be summarized as follows. The electron acceleration mechanisms related to steady reconnection at the X-line as well as due to large-scale flow braking because of the interaction with the Earth's dipolar magnetic field are not sufficient to explain the measurements. Our observations of strong and localized peaks of energetic electron fluxes are due to localized betatron and Fermi acceleration during unsteady reconnection. For the purpose of comparison, we examined a quasi-steady reconnection event discovered in the solar wind ${ }^{12}$ and found that the acceleration of electrons is absent (not shown). Our findings strongly suggest that the very strong acceleration observed during substorms $s^{30}$ and impulsive solar flares ${ }^{31}$ occurs during unsteady reconnection.

Pitch-angle distribution information is crucial to understand the acceleration of energetic electrons during reconnection. This information can only be revealed by in situ space measurements, but not by laboratory or astronomical observations. In addition to the adiabatic acceleration proposed in this letter, non-adiabatic processes may be important in some reconnection events ${ }^{9,32}$. The NASA spacecraft mission scheduled for launch in 2014 will improve the understanding of this issue.

\section{Methods}

We assume the measurement before the jet (at 07:00:18 UT) to be the electron population before reconnection (source, Fig. 3b). Starting from the source, modelling of the electron acceleration during the jet speed rising (Fig. 3c,e) and jet speed falling (Fig. 3d,f) can be done (see ref. 24 for details). In Fig. 3c-g, the factors $f_{\|}$and $f_{\perp}$ are obtained when the modelling results best fit the observations. They are defined as $f_{\|}=\varepsilon_{1 \|} / \varepsilon_{0 \|}$ and $f_{\perp}=\varepsilon_{1 \perp} / \varepsilon_{0 \perp}$, respectively, where the symbols $\varepsilon_{0 \|}$ and $\varepsilon_{1 \|}$ represent the parallel energies of electrons before $\left(\varepsilon_{0 \|}\right)$ and after $\left(\varepsilon_{1 \|}\right)$ the parallel acceleration, and $\varepsilon_{0 \perp}$ and $\varepsilon_{1 \perp}$ are the perpendicular energies of electrons before $\left(\varepsilon_{0 \perp}\right)$ and after $\left(\varepsilon_{1 \perp}\right)$ the perpendicular acceleration.

Received 8 November 2012; accepted 9 May 2013; published online 23 June 2013

\section{References}

1. Shibata, K. et al. Hot-plasma ejections associated with compact-loop solar flares. Astrophys. J. 451, L83-L85 (1995). 
2. Hurley, K. et al. An exceptionally bright flare from SGR 1806-20 and the origins of short-duration g-ray bursts. Nature 434, 1098-1103 (2005).

3. Nagai, T. et al. Structure and dynamics of magnetic reconnection for substorm onsets with Geotail observations. J. Geophys. Res. 103, 4419-4440 (1998).

4. Angelopoulos, V. et al. Tail reconnection triggering substorm onset. Science 321, 931-935 (2008).

5. Pare, V. K. in Magnetic Reconnection in Space and Laboratory Plasmas (ed. Hones, E. W.) 341-346 (Geophysics Monograph 30, American Geophysical Union, 1984).

6. Katz, N. et al. Laboratory observation of localized onset of magnetic reconnection. Phys. Rev. Lett. 104, 255004 (2010).

7. Imada, S. et al. Energetic electron acceleration in the downstream reconnection outflow region. J. Geophys. Res. 112, A03202 (2007).

8. Chen, L. J. et al. Observation of energetic electrons within magnetic islands. Nature Phys. 4, 19-23 (2008).

9. Retinò, A. et al. Cluster observations of energetic electrons and electromagnetic fields within a reconnecting thin current sheet in the Earth's magnetotail. J. Geophys. Res. 113, A12215 (2008).

10. Øieroset, M. et al. Direct evidence for a three-dimensional magnetic flux rope flanked by two active magnetic reconnection X lines at Earth's magnetopause. Phys. Rev. Lett. 107, 165007 (2011).

11. Gosling, J. T. et al. Absence of energetic particle effects associated with magnetic reconnection exhausts in the solar wind. Geophys. Res. Lett. 32, L14113 (2005).

12. Phan, T. D. et al. A magnetic reconnection X-line extending more than 390 Earth radii in the solar wind. Nature 439, 175-178 (2006).

13. Lesch, H. \& Reich, W. The origin of monoenergetic electrons in the Arc of the Galactic Center-Particle acceleration by magnetic reconnection. Astr. Astrophys. 264, 493-499 (1992).

14. Holman, G. D. Acceleration of runaway electrons and Joule heating in solar flares. Astrophys. J. 293, 584-594 (1985).

15. Pritchett, P. L. Relativistic electron production during guide field magnetic reconnection. J. Geophys. Res. 111, A10212 (2006).

16. Egedal, J. et al. Large-scale electron acceleration by parallel electric fields during magnetic reconnection. Nature Phys. 8, 321-324 (2012).

17. Egedal, J. et al. Cause of super-thermal electron heating during magnetotail reconnection. Geophys. Res. Lett. 37, L10102 (2010).

18. Drake, J. F. et al. Electron acceleration from contracting magnetic islands during reconnection. Nature 443, 553-556 (2006).

19. Oka, M. et al. Electron acceleration by multi-island coalescence. Astrophys. J. 714, 915-926 (2010)

20. Hoshino, M. Stochastic particle acceleration in multiple magnetic islands during reconnection. Phys. Rev. Lett. 108, 135003 (2012).

21. Sitnov, M. I. et al. Dipolarization fronts as a signature of transient reconnection in the magnetotail. J. Geophys. Res. 114, A04202 (2009).
22. Lakhina, G. S. Third International Conference on Substorms 23-30 (1996).

23. Ashour-Abdalla, M. et al. Observations and simulations of non-local acceleration of electrons in magnetotail magnetic reconnection events. Nature Phys. 7, 360-365 (2011).

24. Fu, H. S. et al. Fermi and betatron acceleration of suprathermal electrons behind dipolarization fronts. Geophys. Res. Lett. 38, L16104 (2011).

25. Hoshino, M. et al. Suprathermal electron acceleration in magnetic reconnection. J. Geophys. Res. 106, 25979-25997 (2001).

26. Fu, H. S. et al. Pitch angle distribution of suprathermal electrons behind dipolarization fronts: A statistical overview. J. Geophys. Res. 117, A12221 (2012).

27. Lakhina, G. S. A kinetic theory of driven reconnection in the Earth's magnetotail. J. Geophys. Res. 97, 2961-2972 (1992).

28. Daughton, W. et al. Fully kinetic simulations of undriven magnetic reconnection with open boundary conditions. Phys. Plasmas 13, 072101 (2006).

29. Kowal, G. et al. Numerical tests of fast reconnection in weakly stochastic magnetic fields. Astrophys. J. 700, 63-85 (2009).

30. Baker, D. N. et al. High-resolution energetic particle measurements at $6.6 R_{\mathrm{E}}$ 3. Low-energy electron anisotropies and short-term substorm predictions. J. Geophys. Res. 83, 4863-4868 (1978).

31. Lin, R. P. et al. RHESSI observations of particle acceleration and energy release in an intense solar $\gamma$-ray line flare. Astrophys. J. 595, L69-L76 (2003).

32. Khotyaintsev, Y. V. et al. Plasma jet braking: Energy dissipation and nonadiabatic electrons. Phys. Rev. Lett. 106, 165001 (2011).

\section{Acknowledgements}

We thank the Cluster Active Archive for providing the data for this study. Fruitful discussions with P. L. Pritchett and other members of the team 'Particle Acceleration at Plasma Jet Fronts in the Earth's Magnetosphere' at the ISSI are appreciated. This research is supported by the Swedish Research Council under grants 2007-4377, 2009-3902 and 2009-4165. H.S.F. is in part supported by NSFC Grant 40931054 and 973 program 2011CB811404.

\section{Author contributions}

H.S.F. conducted the majority of the data processing, analysis and writing for this study. Y.V.K., A.V., A.R. and M.A. gave suggestions on the unsteady reconnection concept, and participated in the interpretation of the data and the preparation of the figures. A.V. and Y.V.K. developed the software for the data analysis. All the authors discussed the results and commented on the paper.

\section{Additional information}

Reprints and permissions information is available online at www.nature.com/reprints. Correspondence and requests for materials should be addressed to H.S.F.

\section{Competing financial interests}

The authors declare no competing financial interests. 